\title{
Influence of Low Callisto Orbit design on gravity field recovery
}

W. Desprats (1), D. Arnold (1), M. Blanc (2), S. Bertone (3),

A. Jäggi (1), M. Li (4), L. Li (4), and O. Witasse (5)

(william.desprats@aiub.unibe.ch)

- Introduction \& Background

- Methodology \& Results

\begin{tabular}{cc}
\hline Orbit characteristics & Max. degree \\
\hline $88^{\circ} 200 \times 200 \mathrm{~km}$ & 70 \\
$88^{\circ} 400 \times 400 \mathrm{~km}$ & 45 \\
$112^{\circ} 400 \times 400 \mathrm{~km}$ (SSO) & 18 \\
$88^{\circ} 400 \times 1400 \mathrm{~km}$ & 19 \\
\hline
\end{tabular}

- Discussion \& Conclusions

Maximum spherical harmonic degree of

gravity field recoverable for low Callisto

orbits, with different inclinations and

- Acknowledgements \& References

(1) Astronomical Institute, University of Bern, Switzerland, (2) IRAP, Toulouse, France,

(3) University of Maryland, Baltimore County, USA, (4) NSSC, CAS, Beijing, China,

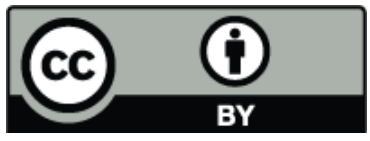

(5) ESTEC, European Space Agency, Noordwijk, The Netherlands. 


\section{Introduction and Background}

- Gan De is a Chinese exploration mission under study, that would fly to Jupiter in the 2030's [3]. An orbiter would be injected into a Low Callisto Orbit to perform an extensive characterization of its surface and interior, investigate its degree of differentiation and search for the possible existence of an internal ocean.

- After an extended tour of the Jupiter system, a first polar elliptic orbit is foreseen for capture around Callisto. Then two polar circular orbits could be used for science investigation. A first one for at least 6 months, and a second one with lower altitude, with the possibility of regular manoeuvres to counteract orbit decay.

- Here, more specific orbits are also investigated due to their relevance for mission design:

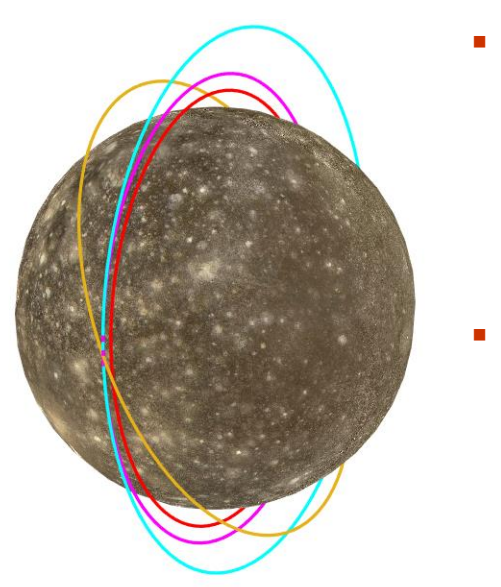

- Sun synchronous orbits (SSO): constant angle between Sun and orbital plane, but with an important polar gap and highly dependent on the gravity field knowledge at low altitude.

- Repetitive Ground Track Orbits (RGTO): defined by an integer triplet $(N, P, Q)[4]$, fixed phase grid defined for $\mathrm{N}^{*} \mathrm{P}+\mathrm{Q}$ orbit revolutions during $\mathrm{P}$ Callisto days [5].

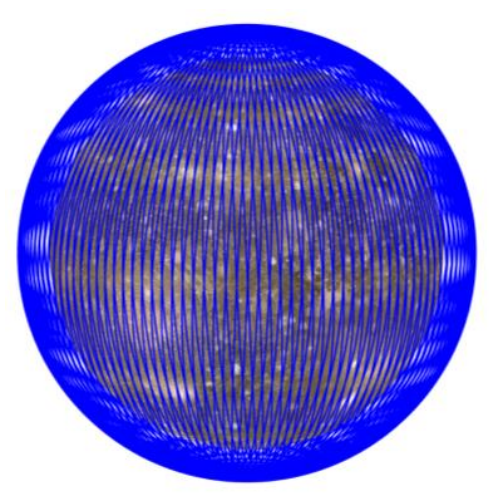

- Orbit propagations in a full force model, as well as the whole gravity field recovery process were done using a development version of the Bernese GNSS Software [6]. 


\section{Set of orbits and simulation setup}

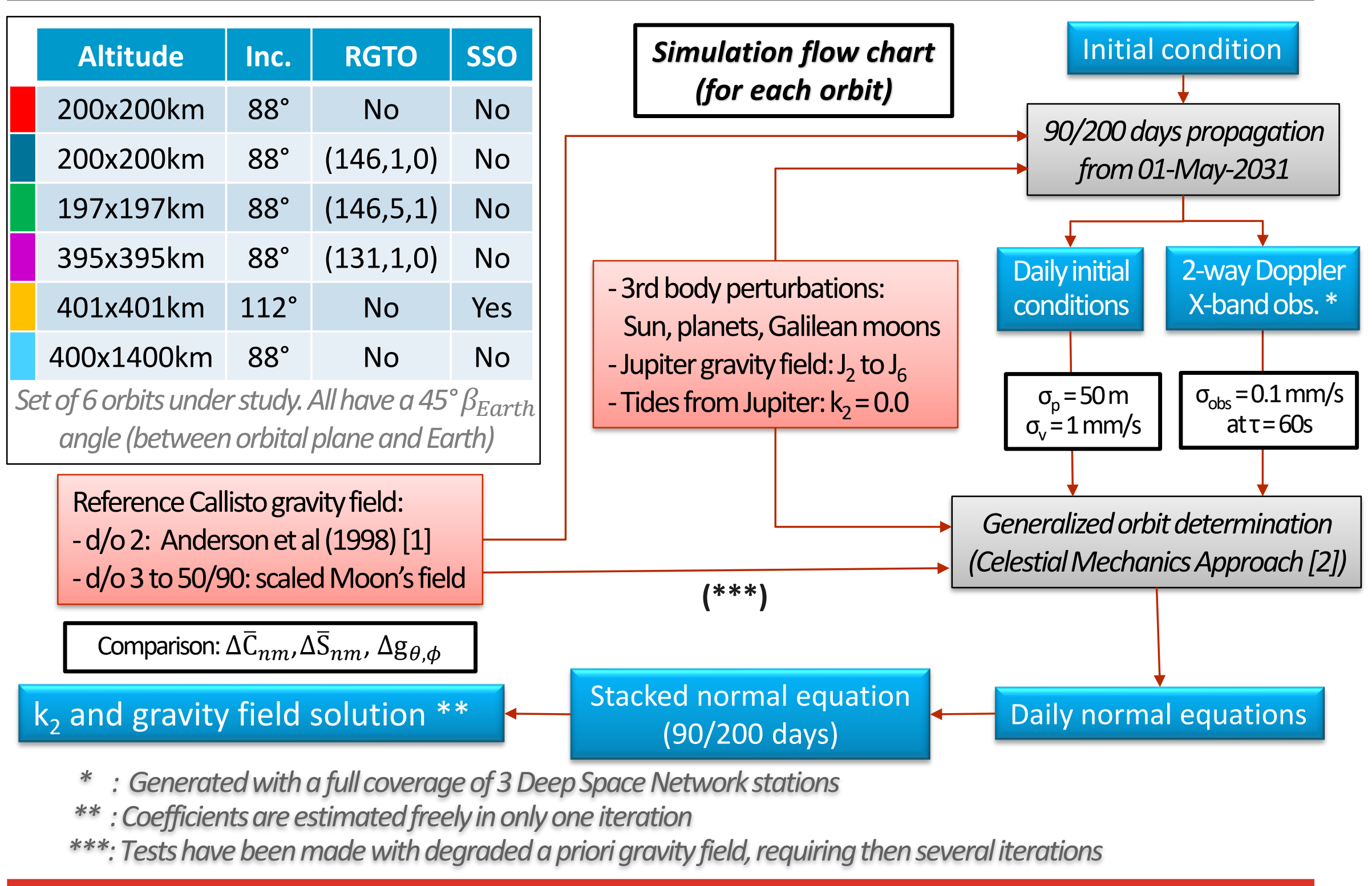




\section{Gravity field recovery}

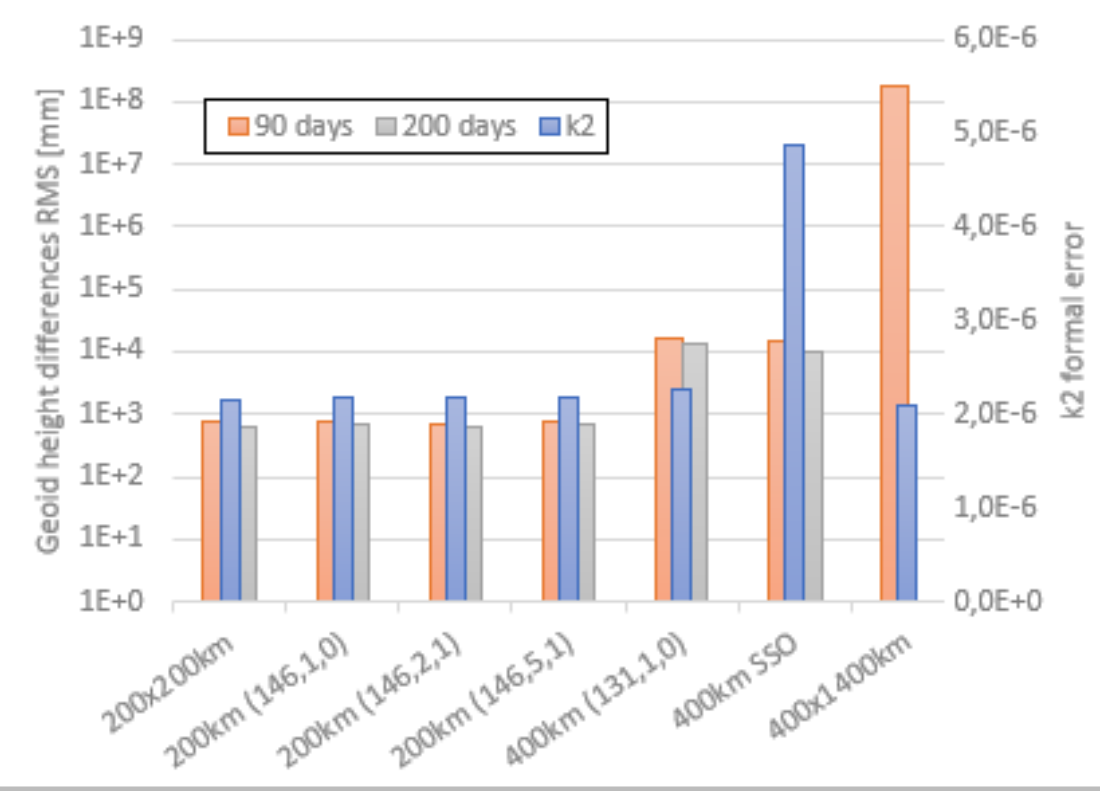

Weighted RMS of geoid height differences $\Delta \mathrm{g}_{\theta, \phi}$ for $90 / 200$ days $\left(\sqrt{\frac{\sum_{\theta, \phi} \cos (\theta) \Delta \mathrm{g}_{\theta, \phi}^{2}}{\text { gridsize }}}\right)$ and $k_{2}$ Love number formal error for 90 days mission computed using an a priori d/o 50 field.

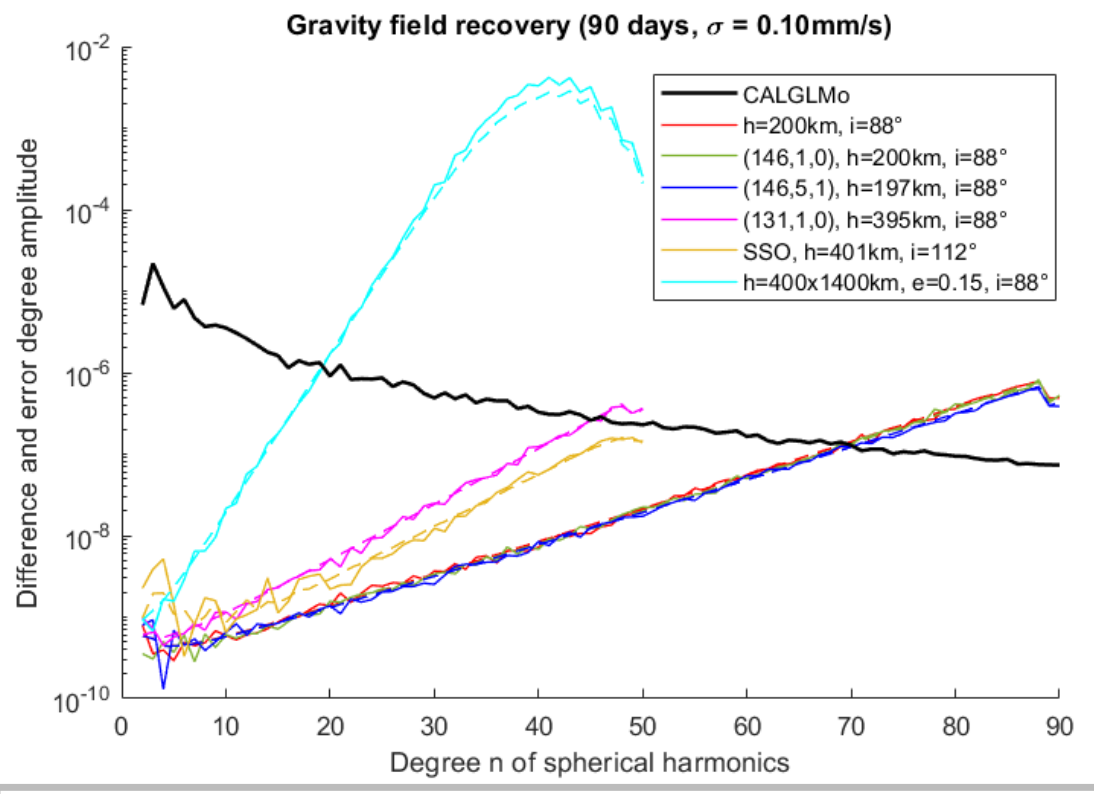

Difference (solid) and error(dashed) degree amplitudes $\left(\mathrm{M}_{\mathrm{n}}=\sqrt{\left.\frac{\sum_{\mathrm{m}=2}^{\mathrm{n}}\left(\Delta \overline{\mathrm{C}}_{\mathrm{nm}}^{2}+\Delta \overline{\mathrm{S}}_{\mathrm{nm}}^{2}\right)}{2 \mathrm{n}+1}\right)}\right.$. For $200 \mathrm{~km}$ orbits, the gravity field was estimated up to $d / 090$

- $22^{\circ}$ polar gap is omitted for the Sun Synchronous Orbit

- With face-on orbit, the gravity field recovery is worse. As an example, the $(146,1,0)$ orbit leads to a larger weighted RMS of geoid height difference for $\beta_{\text {Earth }}=90^{\circ}(153 \mathrm{~cm})$ than for $\beta_{\text {Earth }}=45^{\circ}(88 \mathrm{~cm})$.

- Using a d/o 40 truncated gravity field with the $200 \mathrm{~km}(146,1,0)$ orbit, 4 iterations on the gravity field solution are needed to reach the solution computed with a full d/o 50 a priori gravity field. 


\section{Conclusions}

- A highly eccentric orbit over a time span of 90 days can already improve the knowledge of Callisto's gravity field (up to d/o 19 for a 400x1400km orbit). However, as the eccentricity increases significantly with time, such an orbit is not stable for more than 3 months.

- Sun synchronous orbits suffer from a large polar gap, the recovery of zonal coefficient is then largely impacted, just as Love number $k 2$ recoverability.

- For all non-Sun synchronous orbits, $\beta_{\text {Sun }}$ does not vary much (max. $1.2 \%$ month). A SSO for maximum illumination might then not be compulsory.

- Low altitude polar orbits are the best suited for gravity field recovery. At $400 \mathrm{~km}$ altitude, one can expect to recover the gravity field up to d/o 45 after 90 days.

- Lower orbits are even more beneficial, but will require manoeuvres to increase the orbit lifetime. Repetitive Ground Track Orbits are well suited to efficiently plan station keeping manoeuvres.

- For $200 \mathrm{~km}$ polar orbits a sensitivity up to d/o 70 was found after 90 days. In the case of Callisto, the effect of low density ground tracks (for RGTO) is negligible. 


\section{Acknowledgements \& References}

\section{Acknowledgments}

This study has been funded with the support of the Swiss National Foundation (SNF).

\section{References}

- [1] Anderson, J. D., et al. "Shape, mean radius, gravity field, and interior structure of Callisto." Icarus 153.1 (2001): 157-161.

- [2] Beutler, G., et al. (2010). The celestial mechanics approach: theoretical foundations. Journal of Geodesy, 84(10), 605-624.

- [3] Blanc, M. et al: Gan De: Science Objectives and Mission Scenarios for China's Mission to the Jupiter System, EGU General Assembly 2020, Online, 4-8 May 2020, EGU2020-20179.

- [4] Carrou, Jean-Pierre et al. (1995). Space flight dynamics Volumes 1 et 2

- [5] Cinelli, M. et al: "Polynomial equations for science orbits around Europa." Celestial Mechanics and Dynamical Astronomy 122.3 (2015): 199-212.

- [6] Dach, R. et al. "Bernese GNSS software version 5.2." (2015). 